\title{
Portal Hypertension and a Stiff Liver
}

Felicia D'Alitto ${ }^{1}$, Amina Scherz ${ }^{2}$, Cristina Margini ${ }^{1}$, Hendrik Von Tengg-Kobligk ${ }^{3}$, Matteo Montani ${ }^{4}$, Thomas Pabst ${ }^{5}$, Annalisa Berzigotti ${ }^{6}$

1. Swiss Liver Center, Hepatology, University Clinic for Visceral Surgery and Medicine, Inselspital, University of Bern, Switzerland 2. Department of Medical Oncology, Inselspital, University of Bern, Switzerland 3. Department of Diagnostic, Interventional and Pediatric Radiology, Inselspital, University of Bern, Switzerland 4. Institute of Pathology, University of Berne, Switzerland 5. Department of Medical Oncology, Inselspital, University of Bern, Switzerland, USA 6. Hepatology, University Clinic for Visceral Surgery and Medicine, Inselspital, University of Bern, Bern, CHE

Corresponding author: Annalisa Berzigotti, annalisa.berzigotti@insel.ch

\section{Abstract}

Portal hypertension $(\mathrm{PH})$ is a common clinical syndrome leading to severe complications. In the western world, about $90 \%$ of cases of PH are due to liver cirrhosis, and thanks to the availability of ultrasound elastography methods, this diagnosis is usually confirmed at bedside. We report a case of a patient presenting with PH and ascites initially suspected of suffering from liver cirrhosis. The finding of large hepatomegaly and a massive increase in liver stiffness prompted us to perform a liver biopsy. This revealed no fibrosis, but diffuse primary amyloidosis (AL amyloidosis). We discuss the diagnostic and treatment of this case, with emphasis on non-invasive imaging methods available for diagnosis and follow up.

Categories: Internal Medicine, Radiology, Gastroenterology

Keywords: liver cirrhosis, amyloidosis, multiple myeloma, liver biopsy, elastography, hvpg

\section{Introduction}

Portal hypertension $(\mathrm{PH})$ is a common syndrome featuring severe clinical complications such as ascites and gastrointestinal bleeding due to gastroesophageal varices rupture. The most frequent cause of $\mathrm{PH}$ in the Western world is liver cirrhosis, which accounts for over $90 \%$ of cases. In patients presenting with signs of $\mathrm{PH}$, ultrasound and ultrasound elastography are usually the first diagnostic tests performed, given their high sensitivity and specificity for the diagnosis of cirrhosis [1-2]. In particular, a high liver stiffness is considered an accurate sign of cirrhosis in the context of PH [1-2]. However, a non-expert interpretation of noninvasive diagnostic tests can lead to incorrect diagnosis, and the integration of invasive and non-invasive methods is key for an accurate assessment of this clinical syndrome in order to diagnose rarer causes. Here, we report a clinical case of a patient presenting with PH and increased liver stiffness, initially considered associated with liver cirrhosis but subsequently diagnosed with primary systemic amyloidosis in the context of multiple myeloma. We describe and discuss the use of invasive and non-invasive tests to achieve the final diagnosis and to follow-up the patient.

Received 05/07/2018

Review began 05/13/2018

Review ended 06/06/2018

Published 06/08/2018

๑) Copyright 2018

D'Alitto et al. This is an open access

article distributed under the terms of the

Creative Commons Attribution License

CC-BY 3.0., which permits unrestricted

use, distribution, and reproduction in any medium, provided the original author and source are credited.

\section{Case Presentation}

A 60-year-old man was referred to our center in October 2015 due to ascites and increased liver enzymes ongoing since six months (Table 1). 


\section{Cureus}

\begin{tabular}{|c|c|c|c|}
\hline & Baseline & After 18 months (ongoing VGPR) & Reference normal values \\
\hline Hemoglobin (g/L) & 125 & 121 & $135-168$ \\
\hline Hematocrit & 0.38 & 0.37 & $0.40-0.50$ \\
\hline MCV (fL) & 105 & 91 & $80-98$ \\
\hline Leucocytes (G/L) & 6.6 & 4.96 & $3.5-10.5$ \\
\hline Platelets (G/L) & 222 & 148 & $140-380$ \\
\hline Plasma Creatinine $(\mu \mathrm{mol} / \mathrm{L})$ & 93 & 101 & $59-104$ \\
\hline eGFR according to CKD-EPI (mL/min) & 77 & 68 & $>59$ \\
\hline Sodium (mmol/L) & 140 & 143 & $136-145$ \\
\hline Potassium (mmol/L) & 3.9 & 4.4 & $3.5-4.5$ \\
\hline Plasma protein (g/L) & 58 & 70 & $64-83$ \\
\hline Albumin (g/L) & 32 & 40 & $35-52$ \\
\hline $\lg G(g / L)$ & 7.12 & 7.41 & $7.00-16.00$ \\
\hline Total Bilirubin $(\mu \mathrm{mol} / \mathrm{L})$ & 20 & 5 & $<17$ \\
\hline ASAT (U/L) & 75 & 34 & $<50$ \\
\hline ALAT (U/L) & 35 & 33 & $<50$ \\
\hline Alk. Phosphatase (U/L) & 181 & 65 & $40-130$ \\
\hline Glutamyl transferase (U/L) & 244 & 50 & $<60$ \\
\hline INR ratio & 1.31 & $<1.0$ & \\
\hline Quick (\%) & 57 & $>100$ & $70-130$ \\
\hline
\end{tabular}

TABLE 1: Laboratory tests on admission and after 18 months

VGPR: very good partial response.

He had no previous history of liver disease and he complained of fatigue and weight loss $(16 \mathrm{Kg})$ over eight months. The patient referred alcohol consumption of about $50 \mathrm{~g} /$ day in the last 30 years, which he stopped on the month before admission when ascites was noted. He had no history of chronic diseases except a known allergy to eggs and soy proteins.

On the examination at our center, the patient featured sarcopenia and ascites. Liver ultrasound showed a large hepato-splenomegaly with an irregular surface, a hyperechoic liver parenchyma, and signs of intrahepatic portal hypertension (patent paraumbilical vein; reversed portal venous flow) as well as ascites (Figure 1). No focal liver lesions were observed. 


\section{Cureus}

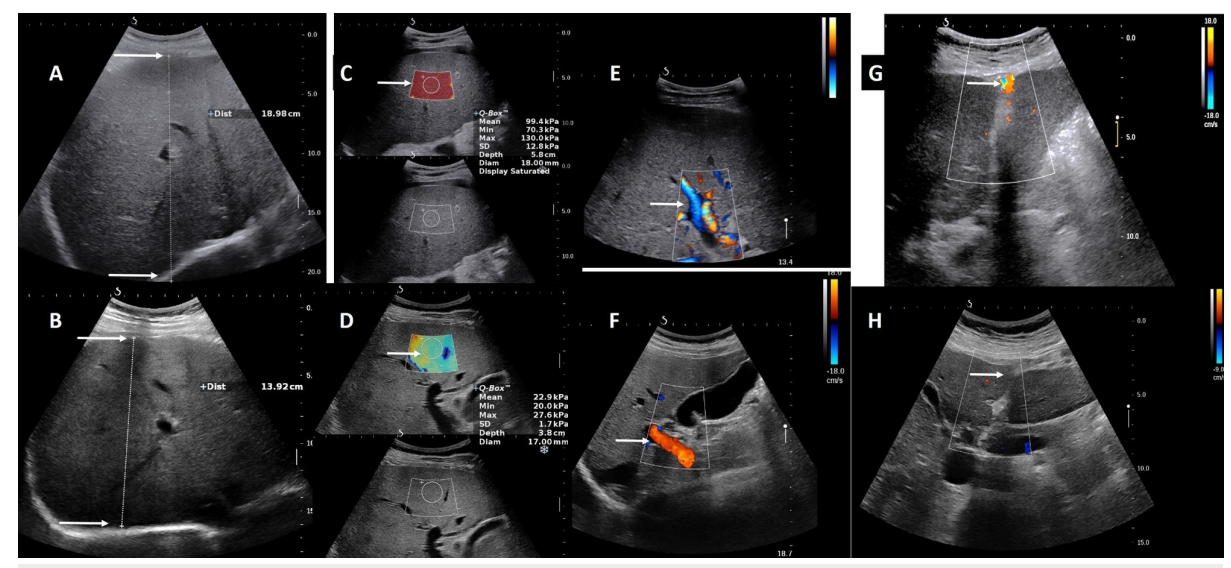

FIGURE 1: Ultrasound and 2D-SWE findings at presentation (October 2015, upper row) and 18 months after diagnosis (ongoing VGPR to chemotherapy; March 2017, lower row)

The antero-posterior diameter of the right liver lobe measured at the mid-clavicular line was markedly enlarged on presentation (Panel A, arrow) and decreased on VGPR (Panel B, arrow). Similarly, liver stiffness by 2D-SWE was very high on presentation (Panel C, arrow) and decreased markedly on VGPR (Panel D, arrow). Intrahepatic portal blood flow was reversed (hepatofugal) on presentation (Panel E, arrow) and returned to normality (hepatopetal) on VGPR (Panel F, arrow). In addition patency of paraumbilical vein was noted on presentations (Panel G, arrow), and was no longer seen on VGPR (Panel H, arrow).

2D-SWE: 2-dimensional shear wave elastography; VGPR: very good partial response.

Liver stiffness measurement (LSM) was performed using two different ultrasound elastography techniques: transient elastography (TE); M probe (FibroScan, Echosens, Paris, France), and 2-dimensional shear wave elastography (2D-SWE); SC6-1 probe (Aixplorer ultrasound system, Supersonic Imagine, Aix-en-Provence, France). Both showed very high values, clearly above normality (Table 2) [1].

\begin{tabular}{|c|c|c|c|}
\hline & Technique & Baseline & $\begin{array}{l}\text { After } 18 \text { months } \\
\text { (ongoing VGPR) }\end{array}$ \\
\hline \multirow[t]{2}{*}{$\begin{array}{l}\text { LIVER } \\
\text { STIFFNESS }\end{array}$} & TE (kPa) & $\begin{array}{l}75 \pm 0 \text { Success } \\
\text { Rate } 100 \%\end{array}$ & $\begin{array}{l}35.3 \pm 7.6 \text { Success } \\
\text { Rate } 100 \%\end{array}$ \\
\hline & 2D-SWE (kPa) & $99.4 \pm 12.8$ & $22.9 \pm 1.7$ \\
\hline LIVER SIZE & $\begin{array}{l}\text { Grey scale ultrasound measurement of the antero-posterior diameter } \\
\text { at the right mid-clavicular line }(\mathrm{cm})\end{array}$ & 18.9 & 13.9 \\
\hline \multirow[t]{2}{*}{$\begin{array}{l}\text { SPLEEN } \\
\text { STIFFNESS }\end{array}$} & TE (kPa) & $\begin{array}{l}45 \pm 3.5 \text { Success } \\
\text { Rate } 100 \%\end{array}$ & $\begin{array}{l}37.4 \pm 5.8 \text { Success } \\
\text { Rate } 100 \%\end{array}$ \\
\hline & 2D-SWE (kPa) & N/A & $31.3 \pm 3$ \\
\hline $\begin{array}{l}\text { SPLEEN } \\
\text { SIZE }\end{array}$ & Grey scale ultrasound measurement of the bipolar diameter $(\mathrm{cm})$ & 14.4 & 13.5 \\
\hline
\end{tabular}

TABLE 2: Comparison of liver and spleen stiffness and size during admission and after 18 months

VGPR: very good partial response; TE: transient elastography; 2D-SWE: two dimensional shear wave elastograpy.

Spleen stiffness was measured by TE and demonstrated values compatible with portal hypertension (Table 2) [2]. A computed tomography (CT) scan confirmed the morphological imaging findings shown on ultrasound; CT-based volumetry of the liver and spleen reached high values of $3298 \mathrm{ml}$ and $621 \mathrm{ml}$, respectively (Figure 2). 


\section{Cureus}

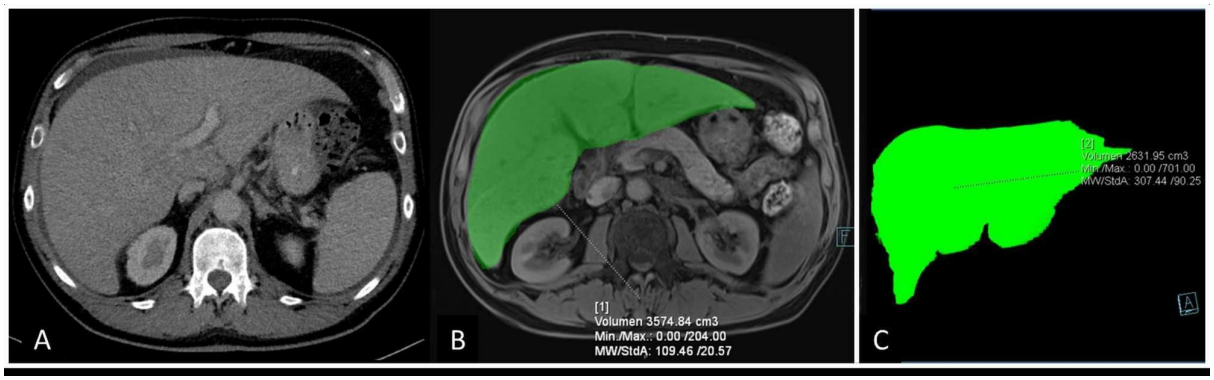

\section{FIGURE 2: Findings on cross sectional imaging on presentation and}

during the follow-up

Computed tomography (CT) scan on presentation showing a clearly enlarged liver (Panel A). Magnetic resonance imaging (MRI) in February 2016 showed a stable hepatomegaly (Panel B), which improved substantially on VGPR one year later (volumetric reconstruction of MRI liver images; Panel C).

VGPR: very good partial response.

Esophagogastroduodenoscopy demonstrated small esophageal varices. A diagnostic paracentesis showed a high serum-ascites albumin gradient ( $2.1 \mathrm{~g} / \mathrm{dL})$, confirming a portal hypertension-related cause. Urinalysis showed a ++ proteinuria. All common causes of chronic liver disease (viral, autoimmune, metabolic) were excluded and a decision to perform liver biopsy was taken. Given the presence of portal hypertension, a transjugular liver biopsy was preferred in order to simultaneously measure the hepatic venous pressure gradient (HVPG) and to minimize the risk of intraperitoneal bleeding.

The procedure was performed by accessing the right jugular vein through Seldinger's technique. The HVPG was $24 \mathrm{mmHg}$ (wedged hepatic venous pressure $33 \mathrm{mmHg}$; free hepatic venous pressure nine $\mathrm{mmHg}$ ), which confirmed a sinusoidal cause of portal hypertension. The liver biopsy was uneventful.

Liver histology showed massive amyloid deposition predominantly in the Dissé space with obliteration of sinusoids and trabecular atrophy, with only minimal portal and perisinusoidal fibrosis (Figures 3-4).

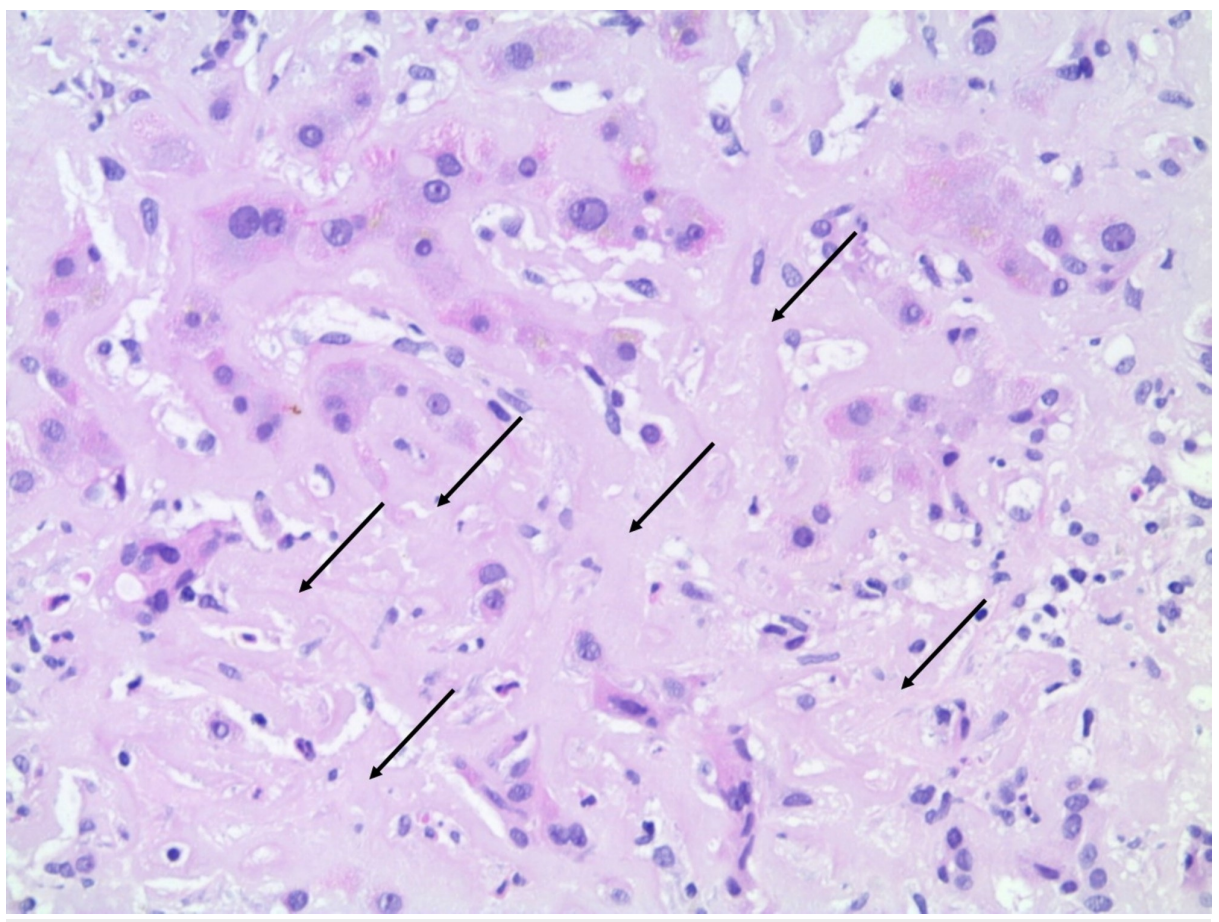

\section{FIGURE 3: Liver histology on diagnosis}

Extensive amyloid deposition with obliteration of sinusoids was observed (arrows). Hematoxylin \& Eosin 200x. 


\section{Cureus}

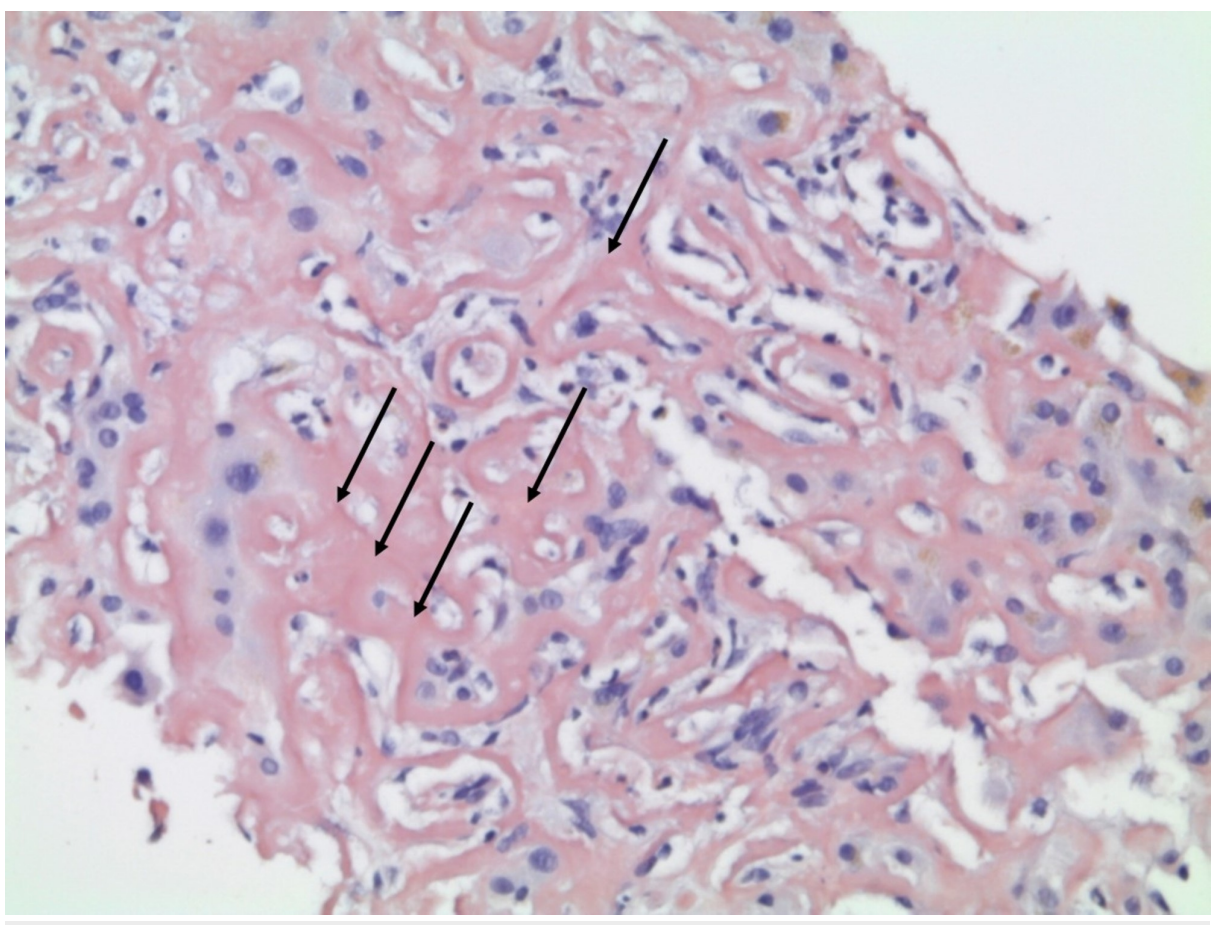

\section{FIGURE 4: Liver histology on diagnosis}

Extensive amyloid deposition with obliteration of sinusoids was observed (arrows). Congo red 100x.

A bone marrow biopsy demonstrated mature cells plasmacellular myeloma with kappa immunophenotype and 20\%-30\% diffuse interstitial infiltration. Congo Red stain showed no amyloid infiltration.

Multiple myeloma with systemic primary amyloidosis (AL) with liver involvement was diagnosed and the patient was referred to the oncology department. Cardiac magnetic resonance imaging (MRI) and electrocardiogram (EKG) did not show features of cardiac involvement. Amyloidosis-related neuropathy affected the autonomous nervous system (relevant orthostatic hypotension) and the peripheral sensory nervous system (hypo- and dysesthesia in the distal upper and lower limbs). Due to the severe malnutrition and sarcopenia associated with amyloidosis and portal hypertension, the patient was put on parenteral nutrition.

From November 26, 2015 until February 23, 2016, the patient was treated with four cycles of a first line regimen according to the CyBorD scheme, comprising cyclophosphamide $350 \mathrm{mg} / \mathrm{m} 2$ intravenous (iv) on days one, eight, and fifteen; $1.0 \mathrm{mg} / \mathrm{m} 2$ bortezomib subcutaneous (sc) on days one, four, eight, and eleven; and dexamethasone $20 \mathrm{mg}$ per os (po) on days one, four, eight, and eleven; within a 21 days schedule.

The treatment was haematologically well tolerated, and orthostatic hypotension resolved completely in the fourth cycle, whereas the patient developed fatigue (CTCAE grade two), loss of appetite, and aggravation of peripheral sensory neuropathy (PNP CTCAE grade two, pain); the latter was assumed as bortezomibtriggered. During the course of chemotherapy, he also developed severe complications of portal hypertension including refractory ascites requiring multiple large-volume paracentesis, bacterial infections, and hepato-renal syndrome.

After the four therapy cycles, the remission status indicated a very good partial response (VGPR), with a difference between involved and uninvolved serum free light chains (DFLC) of $21 \mathrm{mg} / \mathrm{L}$, compared to 258.6 $\mathrm{mg} / \mathrm{L}$ at diagnosis. Abdominal MRI in February 2016 showed stable hepato- (3574 ml) and splenomegaly (613 $\mathrm{ml}$ ) with minimal heterogeneity of the liver parenchyma in the T1w phase suggesting amyloid content.

Few weeks after completion of first line chemotherapy, fatigue and loss of appetite resolved completely; parenteral nutrition could be stopped. A few months later, the drug-induced PNP also improved to CTCAE grade one. The patient refused further consolidation of first-line treatment with high dose chemotherapy (HDCT) and autologous stem cell transplantation (ASCT) because of the side effects related to the induction therapy with CyBorD he previously experienced. No further episodes of clinical decompensation of liver disease were observed.

One year later (eighteen months after the diagnosis), the patient was in very good clinical condition, 
asymptomatic, without ascites and his liver tests improved (Table 1). On ultrasound, the liver was significantly smaller, with slightly heterogenous aspect of the parenchyma as seen in Figure 1 . No signs of portal hypertension were observed; notably, the previously noted paraumbilical vein had disappeared, and the direction of blood flow in the portal vein was now normal (Figure 1). No ascites was present. Both liver and spleen stiffness decreased markedly (Table 2). The MRI based volumetry of liver showed a marked improvement of hepatomegaly ( $2631 \mathrm{ml}$, decrease of $26 \%$ vs. baseline) as seen in Figure 2 and a slight improvement of splenomegaly ( $554 \mathrm{ml}$, decrease of $9.6 \% \mathrm{vs}$. baseline) correlating with the ultrasound and clinical findings. Another year later, his clinical conditions are fully stable.

\section{Discussion}

Amyloidosis is characterized by the abnormal extracellular accumulation of proteinaceous fibrillary material (amyloid) that is fairly resistant to normal proteolytic digestion. Systemic amyloidosis is subclassified by a particular precursor protein into two major forms, AL amyloidosis and secondary amyloidosis (AA amyloidosis) [3]. The primary form is characterized by amyloid deposits composed of the light chains fragment of immunoglobulin and it is associated with B-cell dyscrasia, particularly multiple myeloma, and monoclonal gammopathy. The secondary form is characterized by the deposition of an amyloid A protein (non-immunoglobulin) and it follows or co-exists with various disorders such as rheumatoid arthritis, tuberculosis, chronic long-standing infections or malignant tumors, particularly renal cell carcinoma [3].

The liver is a common site of amyloid deposition in primary AL amyloidosis. In an autopsy series [4], 70\% of the patients with primary systemic amyloidosis had liver involvement, which is, however, most of the times asymptomatic. There are few reported cases of portal hypertension due to primary systemic amyloidosis [56]. Notably, liver involvement is associated with a reduced survival in patients with primary systemic amyloidosis (mean survival time: 10 to 14 months vs. 12 to 18 months in patients without liver involvement) [7-9] and the presence of portal hypertension further worsens patients' outcome (mean survival time: eight to nine months) [10].

As in any severe disease, an early recognition of primary amyloidosis is crucial and liver involvement should be diagnosed for further risk stratification. Liver involvement can be suspected based on imaging findings, but its confirmation requires a liver biopsy. However, a higher risk of bleeding in patients with amyloidosis has been reported [11]. This has been ascribed to different factors: a) reduced synthesis and/or malabsorption of vitamin K-dependent clotting factors resulting in coagulopathy; b) rapid clearance of factor X due to binding to amyloid fibrils, resulting in a deficiency of factor X [12]; c) decreased contractility of vessels infiltrated by amyloid, which, once lacerated, may not clot normally [13-15].

Transjugular liver biopsy is a safe alternative in case of increased bleeding risk [16]. In our patient, this route was chosen before suspecting the diagnosis of amyloidosis due to the concomitant presence of portal hypertension.

In our patient, the finding of an increased alcohol consumption initially suggested a diagnosis of cirrhosis. This was questioned due to the presence of large hepatomegaly and abnormally high liver stiffness measurement. Interestingly, 2D-SWE (a modern ultrasound elastography technique which provides B-mode imaging in addition to elasticity measurements, and which can be performed in patients with ascites) [1] demonstrated extremely high values (mean $99.4 \mathrm{kPa}$ ), which are compatible with, but not typical of cirrhosis (usually not exceeding 70-75 kPa). Two previous cases have reported extremely high values of liver stiffness measured either with TE or with SWE [17-18], similar to the ones observed in our patient.

Our case is the first demonstrating that a VGPR to chemotherapy, likely decreasing liver amyloid content, was associated to a decrease in liver stiffness measured by TE and 2D-SWE, and was accompanied by a disappearance of signs of portal hypertension. Notably, liver stiffness improved but remained higher than normal (and in a range that could be still compatible with cirrhosis), but spleen stiffness measurement (SSM) completely normalized after treatment. SSM is increasingly recognized as a sensitive and specific noninvasive surrogate of portal pressure [2], with improved accuracy as compared to LSM.

\section{Conclusions}

We have described the case of a patient presenting with ascites due to portal hypertension. We report how non-invasive diagnostic methods helped in the characterization of the cause of the syndrome. The presence of a very high liver stiffness and of patency of the paraumbilical vein suggested an intrahepatic cause of the syndrome. However, some characteristics such as a large hepatomegaly and an exceedingly high value of liver stiffness were not typical of cirrhosis (the most common cause of intrahepatic portal hypertension). Liver biopsy was determinant in diagnosing primary amyloidosis, and this underlines how non-invasive and invasive diagnostic methods provide complementary information in difficult cases. After the identification of hepatic amyloidosis, our patient was diagnosed with multiple myeloma and underwent chemotherapy. He showed a VGPR to treatment, and the clinical complications of portal hypertension disappeared. In parallel, non-invasive signs of the syndrome improved. This case, whose good outcome is not expected (primary amyloidosis presenting with portal hypertension usually has an ominous prognosis) shows that imaging and liver and spleen stiffness are useful techniques to be used in patients with portal hypertension on diagnosis 
and might be used as non-invasive biomarkers to follow-up patients with liver involvement due to primary systemic amyloidosis.

\section{Additional Information \\ Disclosures}

Human subjects: Consent was obtained by all participants in this study. Cantonal Ethics Committee (KEK) Bern issued approval N/A. N/A: this is a case report and the patient provided a written informed consent. Conflicts of interest: In compliance with the ICMJE uniform disclosure form, all authors declare the following: Payment/services info: All authors have declared that no financial support was received from any organization for the submitted work. Financial relationships: All authors have declared that they have no financial relationships at present or within the previous three years with any organizations that might have an interest in the submitted work. Other relationships: All authors have declared that there are no other relationships or activities that could appear to have influenced the submitted work.

\section{References}

1. Dietrich CF, Bamber J, Berzigotti A, et al.: EFSUMB guidelines and recommendations on the clinical use of liver ultrasound elastography, update 2017 (Long Version). Ultraschall in Med. 2017, 38:e16-e47. 10.1055/s0043-103952

2. Berzigotti A: Non-invasive evaluation of portal hypertension using ultrasound elastography . J Hepatol. 2017, 67:399-411. 10.1016/j.jhep.2017.02.003

3. Falk RH, Comenzo RL, Skinner M: The systemic amyloidoses. N Engl J Med. 1997, 337:898-909. 10.1056/NEJM199709253371306

4. Iwata T, Hoshii Y, Kawano H, et al.: Hepatic amyloidosis in Japan: histological and morphometric analysis based on amyloid proteins. Hum Pathol. 1995, 26:1148-1153.

5. Kobayashi T, Sato Y, Ichida T, et al.: Primary amyloidosis with liver failure and acute renal failure treated with emergency living-related liver transplantation: a case report. Transplant Proc. 2003, 35:356-357.

6. Norero B, Perez-Ayuso RM, Duarte I, et al.: Portal hypertension and acute liver failure as uncommon manifestations of primary amyloidosis. Ann Hepatol. 2014, 13:142-149.

7. Kyle RA, Gertz MA: Primary systemic amyloidosis: clinical and laboratory features in 474 cases . Semin Hematol. 1995, 32:45-59.

8. Tan SY, Pepys MB, Hawkins PN: Treatment of amyloidosis. Am J Kidney Dis. 1995, 26:267-285.

9. Peters RA, Koukoulis G, Gimson A, Portmann B, Westaby D, Williams R: Primary amyloidosis and severe intrahepatic cholestatic jaundice. Gut. 1994, 35:1322-1325.

10. Park MA, Mueller PS, Kyle RA, Larson DR, Plevak MF, Gertz MA: Primary (AL) hepatic amyloidosis: clinical features and natural history in 98 patients. Medicine (Baltimore). 2003, 82:291-298. 10.1097/01.md.0000091183.93122.c7

11. Sucker C, Hetzel GR, Grabensee B, Stockschlaeder M, Scharf RE: Amyloidosis and bleeding: pathophysiology, diagnosis, and therapy. Am J Kidney Dis. 2006, 47:947-955. 10.1053/j.ajkd.2006.03.036

12. Kos CA, Ward JE, Malek K, et al.: Association of acquired von Willebrand syndrome with AL amyloidosis . Am J Hematol. 2007, 82:363-367. 10.1002/ajh.20829

13. Kyle RA, Gertz MA, Greipp PR, et al.: Long-term survival (10 years or more) in 30 patients with primary amyloidosis. Blood. 1999, 93:1062-1066.

14. Ebert EC, Nagar M: Gastrointestinal manifestations of amyloidosis. Am J Gastroenterol. 2008, 103:776-787. 10.1111/j.1572-0241.2007.01669.x

15. Gnann JS, Bressler GS, Bodet CA: Bleeding manifestations in 100 patients with amyloidosis . Intern Med. 1983, 249:1322-1324.

16. Kalambokis G, Manousou P, Vibhakorn S, et al.: Transjugular liver biopsy--indications, adequacy, quality of specimens, and complications--a systematic review. J Hepatol. 2007, 47:284-294. 10.1016/j.jhep.2007.05.001

17. Lanzi A, Gianstefani A, Mirarchi MG, Pini P, Conti F, Bolondi L: Liver AL amyloidosis as a possible cause of high liver stiffness values. Eur J Gastroenterol Hepatol. 2010, 22:895-897. 10.1097/MEG.0b013e3283309d5b

18. Trifanov DS, Dhyani M, Bledsoe JR, et al.: Amyloidosis of the liver on shear wave elastography: case report and review of literature. Abdom Imaging. 2015, 40:3078-3083. 10.1007/s00261-015-0519-4 